OPEN ACCESS

Edited by:

Ulas Sunar,

University at Buffalo, USA

Reviewed by:

David B. Stout,

UCLA Crump Institute for Molecular Imaging, USA

Thomas Foster

University of Rochester, USA

Henry Hirschberg,

University of California, Irvine, USA

*Correspondence:

Conor L. Evans,

Wellman Center for Photomedicine,

Harvard Medical School,

Massachusetts General Hospital, CNY149-3, 13th St, Charlestown MA

02129, USA

evans.conor@mgh.harvard.edu

Specialty section:

This article was submitted to

Biomedical Physics, a section of the

journal Frontiers in Physics

Received: 21 January 2015 Paper pending published:

12 February 2015

Accepted: 04 March 2015

Published: 27 March 2015

Citation:

Evans CL (2015) Three-dimensional in vitro cancer spheroid models for photodynamic therapy: strengths and opportunities. Front. Phys. 3:15 doi: 10.3389/fphy.2015.00015

\section{Three-dimensional in vitro cancer spheroid models for photodynamic therapy: strengths and opportunities}

\author{
Conor L. Evans * \\ Wellman Center for Photomedicine, Harvard Medical School, Massachusetts General Hospital, Boston, MA, USA
}

Three dimensional, in vitro spheroid cultures offer considerable utility for the development and testing of anticancer photodynamic therapy regimens. More complex than monolayer cultures, three-dimensional spheroid systems replicate many of the important cell-cell and cell-matrix interactions that modulate treatment response in vivo. Simple enough to be grown by the thousands and small enough to be optically interrogated, spheroid cultures lend themselves to high-content and high-throughput imaging approaches. These advantages have enabled studies investigating photosensitizer uptake, spatiotemporal patterns of therapeutic response, alterations in oxygen diffusion and consumption during therapy, and the exploration of mechanisms that underlie therapeutic synergy. The use of quantitative imaging methods, in particular, has accelerated the pace of three-dimensional in vitro photodynamic therapy studies, enabling the rapid compilation of multiple treatment response parameters in a single experiment. Improvements in model cultures, the creation of new molecular probes of cell state and function, and innovations in imaging toolkits will be important for the advancement of spheroid culture systems for future photodynamic therapy studies.

Keywords: 3D cultures, spheroids, oxygenation, imaging, advanced microscopy

\section{Introduction}

Optical imaging has enjoyed considerable success in supporting investigations into the myriad of effects induced by anti-neoplastic therapies. From tracking cytotoxic effects induced by chemotherapy [1] to measuring the normalization of blood vessels by vascular endothelial growth factor (VEGF) blockade [2], optical imaging tools have provided quantitative metrics of therapeutic outcomes on the tumoral, cellular, and subcellular scales. In studies of photodynamic therapy, optical tools have been particularly useful in vivo, with the ability to measure not only treatment response but also the three-dimensional uptake of photosensitizers and the location and rate of photobleaching to assess therapeutic efficacy [3]. However, not all tumors lend themselves to optical assessment; only select tumor types can be visualized in clinical settings, and only a handful of cancer types are compatible with window-type methods in animal models [4]. Particularly problematic are metastatic diseases of the peritoneal cavity, including colorectal, ovarian, and pancreatic cancer, which are difficult to visually assess for treatment planning and therapeutic efficacy studies. Monolayer cultures, while useful for studying treatment effects, are too simple to replicate the many heterogeneous treatment effects found in vivo.

Three dimensional tumor models partially address these limitations, enabling long-term studies of unique model tumors and individual cells over time. The 3D model environment can 
be advantageous for treatment studies, as many environmental variables can be controlled and adjusted (e.g., oxygenation, $\mathrm{pH}$ ) as compared to animal models. Spheroid cultures, in particular, are unique systems that replicate many of the features found within tumors in vivo, including cell-cell and cellmatrix interactions. 3D spheroid cultures can grow to recapitulate microenvironmental conditions including the deposition of extracellular matrix, cellular polarization, steep oxygen gradients, acidosis, hypoxia and necrotic cores. These models also offer the advantage of history: they have been in development and use since the 1970's, when nodular cultures were grown in spinner flasks that allowed individual tumor cells to cluster into suspended nodules [5]. Since then, numerous biological and technological advances have been made, enabling more advanced suspension cultures, agar culture systems, cultures based on extracellular matrix deposition, matrigel 3D cultures, hanging drop methods, and synthetic matrix-based cultures [610]. While many multicellular spheroids are monocultures, coculture spheroids have seen extensive use and can model aspects of tumor heterogeneity. With thousands of studies utilizing 3D in vitro models, it is possible to select optimal model systems for any given study.

These features, while useful for general therapeutic experiments, are of particular help in optimizing photodynamic therapy (PDT): as many photosensitizers are fluorescent, optical imaging can be employed to dynamically visualize the uptake and localization of these agents at the cellular and subcellular level. Treatment response, including photobleaching, apoptosis, and necrosis can be tracked in real-time, with spatiospecific monitoring providing links between microenvironmental variables and treatment outcome. In addition, by taking advantage of the high-throughput nature of optical imaging, many thousands of model tumors can be studied in a single experiment, which can allow studies to assess the effect of heterogeneity on treatment outcome.

\section{Spheroid Model Systems for PDT}

Spheroid cultures have served photodynamic research for nearly three decades, $[11,12]$ with the majority of studies utilizing the $3 \mathrm{D}$ in vitro model systems to examine the uptake and therapeutic efficacy of photosensitizers. As it is outside the scope of this minireview to cover every spheroid PDT experiment, a subset of studies focused on imaging-based methods will be used to illustrate the key utilities of 3D spheroid models.

\subsection{Capturing PDT Outcomes with High-Content, High Throughput Imaging Toolkits}

One of the major strengths of 3D in vitro cultures is the ability to simultaneously grow, monitor, and treat many thousands of individual tumor spheroids in a single experiment. As spheroids can be heterogeneous in size, shape, and a host of other factors, large-scale studies across thousands of spheroids can reveal patterns of response, rare events, and important subpopulations. Early studies utilizing spheroids at the Beckman Laser Institute, for example, examined the efficacy of 5-aminolevulinic acid/protoporphyrin IX (ALA/PpIX)-PDT by observing the growth and survival of spheroids following treatment [13-15]. In a study by Hirshberg et al. the effect of different ALA derivatives on the growth and photodynamic therapy outcomes of glioma spheroids was investigated. By observing the diameters and surviving fractions of a large number of spheroids, it was found that the lipophilic esters of ALA, such as benzyl and hexyl ALA, were considerably more effective due to their enhanced uptake within the model tumor nodules [13]. Imaging tools, such as wide field fluorescence and confocal fluorescence microscopy, have more recently enabled the addition of highcontent data collection. Overlay model systems, where spheroids all reside at the same depth in culture, have been particular useful, as automated stages can be used to scan over large fields of view and acquire mosaics of photosensitizer uptake and treatment response. In a study by Celli et al., the Live/Dead stain was applied to ovarian cancer spheroids grown in overlay to study the effects of both carboplatin chemotherapy and benzoporphrin derivative monoacid (BPD)-PDT [16]. Through the acquisition of high-resolution, large area mosaics, this study found the carboplatin therapy was ineffective in killing cells within the center of the model tumor nodules. BPD-PDT, on the other hand, was found to be more uniformly effective through spheroids. Recent advances by Celli, Hasan, and colleagues have developed this high-content imaging toolkit to collect and model a far greater set of treatment parameters $[17,18]$ to support next-generation photodynamic therapy screening experiments (Figure 1).

High-content imaging tools can also be used to follow longterm changes in 3D spheroid cultures over time, providing insight into cell death processes, nodule unpacking, and treatment resistance. Studies in glioma spheroid cultures examined not only PDT efficacy, but also the effects of therapy on the migratory and invasive behavior of individual glioma cells posttreatment; it was observed that both ALA/PpIX and Photofrin photodynamic therapy had the significant effect of suppressing cell migration and invasion in $3 \mathrm{D}$ cultures $[19,20]$. This reduction in migratory activity, which may be of importance in reducing glioma metastasis in vivo, was postulated by Hirshberg et al. to arise from the inhibition of cell-matrix adhesion triggered by PDT [21]. The spatial patterns of apoptosis and structural degradation have additionally been mapped over time with imaging toolkits to select more optimal treatment methods [22-24]. For example, BPD-PDT was recently applied to small $(<250 \mu \mathrm{m}$ diameter) and large ( $>250 \mu \mathrm{m}$ diameter) 3D ovarian cancer spheroids, with the treatment outcome mapped over the course of several days with optical coherence tomography (OCT). While small nodules were found to undergo widespread apoptosis, large nodules were observed to have only peripheral cellular death and continued to grow unabated [22].

\subsection{Mapping Three-Dimensional Photosensitizer Uptake}

These spatial patterns of therapeutic efficacy can result from a host of factors, including the uptake and localization of photosentizers. 3D spheroid cultures, which simulate avascular tumors, have been helpful in modeling the uptake and penetration of photosensitizers through multiple cell layers. For example, studies from the Beckman Laser Institute, focused on the photodynamic 


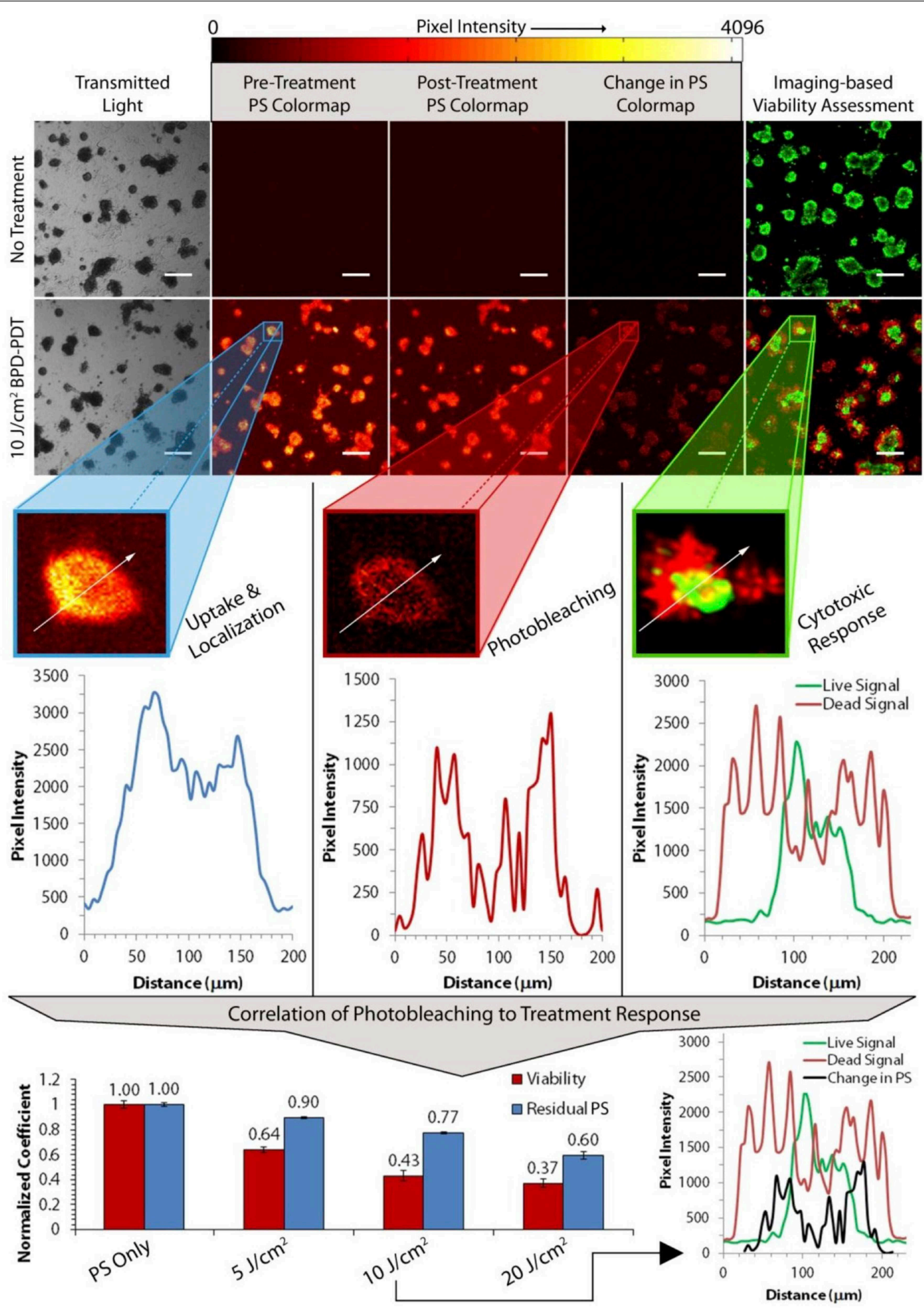

FIGURE 1 | High-throughput imaging methods can provide quantitative insight into PDT treatment parameters and outcomes. High-throughput imaging approaches, such as the method described here by Glidden et al. [17] (reproduced with permission), offer the means to screen tens of thousands of individual tumor nodules and their treatment response parameters in a single experiment. For example, by monitoring the change in photosensitizer fluorescence ("Change in PS" column), one can monitor photosensitizer photobleaching to infer and correlate photosensitizer activity with therapeutic outcome. 

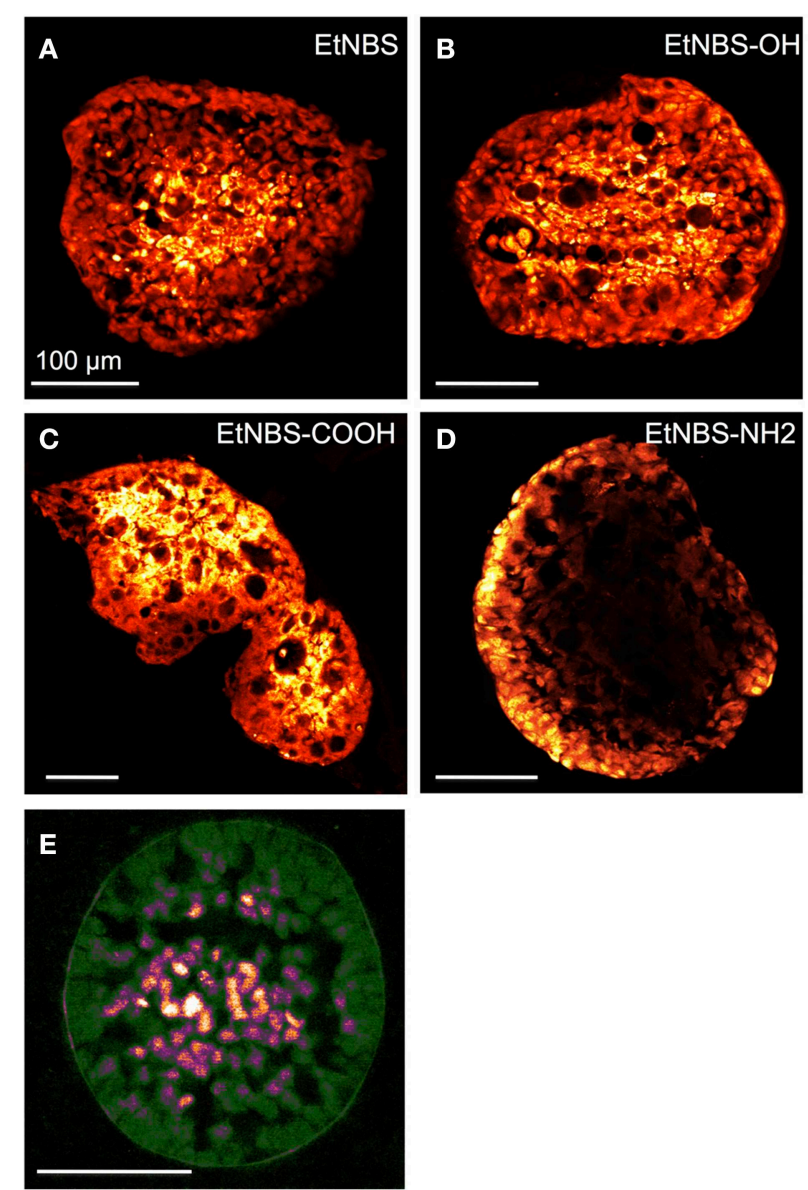

FIGURE 2 | Physical sectioning and fluorescence microscopy can show the uptake and localization patterns of photosensitizers with 3D spheroids. An advantage of spheroid cultures is the ease with which they can be manipulated so as to examine the microscale patterns of photosensitizer uptake. In this figure reproduced from Klein et al. [29], OVCAR5 spheroids were incubated with EtNBS and several of its derivatives for $4.5 \mathrm{~h}$ prior to being frozen and sliced into thin sections. These sections can be readily imaged by confocal microscopy to visualize the differential uptake of the compounds. (A) EtNBS, (B) EtNBS-OH, (C) EtNBS-COOH, and (D) EtNBS-NH2. (E) Two-photon image of a pimodinazole-stained 3D tumor nodule shown here in false color to visualize hypoxic tumor cells contained inside the large nodules. Reprinted with permission from [29]. Copyright 2012 American Chemical Society.

therapy of glioma, have utilized spheroid cultures to explore the uptake and localization of ALA/PpIX-based regimens [25, 26]. Using confocal microscopy, Bigelow et al. similarly investigated the distribution of PpIX in multicellular spherods [27]. Xiao et al., using a spheroid bladder cancer model, examined the uptake distributions of several porphyrin photosensitizers using confocal microscopy. Like other investigations examining porphyrin-based photosensitizers, each molecule was found to localize primarily on the exterior of individual nodules. Interestingly, though both free and liposomal formulations of BPD-MA and hypocrellin R2 (HBBA-R2) were found to localize to the periphery of spheroids, the liposome-delivered photosensitizers demonstrated nearly exclusive peripheral uptake [28]. A study examining the uptake behavior of both the porphyrin BPD-MA and the benzophenothiazinium dye EtNBS in ovarian cancer 3D spheroid cultures found that, while BPD-MA bound to the periphery of nodules, EtNBS completely penetrated and localized to the highly acidic center of spheroids. This advantageous localization pattern likely arose from the cationic small molecule nature of EtNBS, which enables its efficient diffusion toward low $\mathrm{pH}$ gradients [23]. In a follow-up study investigating side chain modifications of EtNBS, 3D ovarian cancer spheroid cultures were used as a model platform to examine the uptake of a small derivative library with confocal microscopy and traditional frozen section preparation. Despite only small modifications to the molecule's side chain, alterations in charge and partition coefficient were found to have major effects on the uptake of the modified photosensitizers (Figure 2) [29].

\subsection{Examining the Effects of Oxygenation on Treatment Outcome}

Spheroid cultures, in which cells are tightly packed into threedimensional layers of cells, serve as excellent models for measuring the effects of oxygen diffusion and consumption, both during normal growth and photodynamic therapy. Notably, many of the first PDT spheroid studies were led by Tom Foster and his colleagues to investigate photosensitizer uptake, localization, and oxygenation effects using combinations of standard imaging tools and oxygen microelectrodes [30-33]. Interestingly, the use of oxygen electrodes demonstrated that steep oxygen gradients exist within spheroidal model tumors, which can have major effects of PDT outcome. As there is an important interplay between oxygen consumption and supply during PDT, this relationship was investigated by exploring fluence-rate effects. The results of this study showed that low fluence-rate PDT optimized the degree of cell killing due to the greater relative abundance of oxygen throughout therapy [34]. In a more detailed investigation of this effect, oxygenation was both spatially and temporally mapped using oxygen electrodes to determine oxygen diffusion and consumption during Photofrin-PDT in EMT6 cell line spheroids [35]. This oxygenation measurement toolkit was recently combined with imaging by Mitra et al., where EMT6 spheroids were utilized in an imaging study examining the efficacy of mTHPC (Foscan) vs. Photofrin. By combining confocal microscopy to measure the uptake and photobleaching of the photosensitizers with oxygenation measurements tracking spheroid oxygen tension $\left(\mathrm{pO}_{2}\right)$, this study gathered a set of experimental parameters that were able to explain mTHPC's enhanced efficacy over Photofrin [36].

Similarly, in studies of ALA/PpIX therapy in glioma spheroids, it was found that the majority of cell killing occurred at the spheroid periphery for both 3 and $6 \mathrm{~J} / \mathrm{cm}^{2}$ doses. As ALA can readily penetrate throughout large $(>250 \mu \mathrm{m})$ spheroids, these effects were proposed to arise from depleted oxygen within the spheroid cores, suggesting that low fluence or repetitive PDT sessions would have greater effect in vivo $[25,26]$. These spheroid size-dependent effects were similarly observed by West and colleagues in studies of human colon adenocarcinoma cultures $[37,38]$. Indeed, studies examining the utility of hyperoxygenation therapy on spheroid cultures found that higher $\mathrm{pO}_{2}$ levels improved the outcome of hypericin PDT [39-41]. 
Hypoxia in the cores of ovarian cancer spheroids was imaged using the 2-nitroimidazole pimodinazole, revealing that a significant fraction of cells within a $3 \mathrm{D}$ spheroid can experience oxygen levels less than $20 \mathrm{mmHg}$, even when the nodule's diameter is less than $200 \mu \mathrm{m}$ (Figure 2). As would be expected, these hypoxic regions also experienced severe acidosis, with extracellular $\mathrm{pH}$ levels measured between 5 and 6 . As expected, the outcome of BPD-PDT to these cultures was found to have a strong size-dependence, with larger nodules experiencing reduced cell killing. These studies suggested that photosensitizers capable of Type-I photochemistry may have advantages within the oxygendepleted spheroid cores. Two studies making use of EtNBS and its derivatives, which operate predominantly through Type-I phototoxicity, found that the cores of large, hypoxic nodules could be effectively eliminated even at low light doses [23, 29]. When EtNBS-PDT was applied to nodule cultures, an inverse sizedependence was observed, with larger spheroids experiencing greater therapeutic efficacy [23].

\subsection{Screening for Combination Regimens}

$3 \mathrm{D}$ spheroid cultures serve as outstanding test beds to examine potential interactions between therapeutics, with the potential to examine not only arrays of different combination of therapies, but also their order and timing. In a study by Hirschberg et al., ALA-PDT was combined with hyperthermia to examine for potential synergism [42]. Interestingly, hyperthermia below $49^{\circ} \mathrm{C}$ was found to have minimal effects in $3 \mathrm{D}$ cultures, even though lower temperatures $\left(44^{\circ} \mathrm{C}\right)$ were found effective in $2 \mathrm{D}$ monolayer cell cultures; this greater resistance to temperature was thought to be the likely result of cell-cell contacts providing pro-survival signals. However, when hyperthermia was combined with PDT, significant synergism was observed. The timing of PDT was additionally found to be important: PDT and hyperthermia were found to have a significant degree of interaction when applied concurrently; this synergy was lost when PDT was applied $24 \mathrm{~h}$ later. While this combination method was found to be synergistic and cause apoptosis, the effect was not widespread and was limited to the periphery of spheroids, likely due to low levels of oxygenation deeper within the model tumors. The same group additionally investigated the potential for synergy between ALAPDT and radiotherapy. While synergy was observed between PDT and gamma irradiation, significant interactions were found only under low light fluence levels. Unlike in the case of hyperthermia, the timing of the two therapies was not found to have an influence [15]. An interesting study exploring the interaction of haematoporphyrin derivative $(\mathrm{HpD})$ with verapamil, a calcium channel blocker, observed that the small molecule improved the overall efficacy of PDT in colorectal cancer spheroids. This beneficial interaction was hypothesized to arise from verapamil's ability to hamper cellular efflux pumps, thus increasing intracellular concentrations of the photosensitizer [43].

A more recent study led by Rizvi et al. utilized high-content imaging assays to explore the combination regiment of carboplatin chemotherapy with BPD-PDT [44]. Carboplatin on its own had previously been found to induce only peripheral cell killing in spheroids, [16] an observation that was thought to arise from the limited penetration of the platinum agent [45]. However, when carboplatin was administered following PDT, the chemotherapy drug was significantly synergistic, leading to widespread cell death in ovarian cancer spheroid cultures. Interestingly, when the order of administration was reversed, no synergism could be observed. Confocal imaging of spheroids treated with BPDPDT revealed a pattern of "unpacking"-type structural degradation, where individual spheroids were observed to have increased space between adjacent cells. It was hypothesized that not only did this unpacking lead to the enhanced uptake of carboplatin into PDT-treated spheroids, but the structural change might have also led to re-oxygenation of the spheroid core, enabling greater platin therapeutic efficacy. An OCT-based study of EtNBS derivatives observed similar in vitro tumor nodule unpacking following PDT, suggesting that the mechanism proposed by Hasan and colleagues might be a general approach to enhancing combination treatments [24].

\section{Limitations and Outlook}

3D in vitro cultures have been highly useful tools for understanding and optimizing photodynamic therapy for cancer. Imaging tools, ranging from simple bright field to more advanced tomographic imaging tools continue to provide new insight into the processes that underlie treatment response behaviors. Despite these advances, new methods for imaging-based quantification of 3D cultures are needed. Many of the imaging assessment methods used in 3D studies have significant limitations that likely lead to under- or overestimation of PDT efficacy in some models. For example, the Live/Dead staining kit is useful when applied to small ( $<250 \mu$ m diameter) spheroids, but suffers from poor penetration and uptake once model tumor nodules grow too large. The same effect has been observed for apoptosis markers, such as ApoTrace, which can only penetrate a few cell layers deep [23]. Without the ability to deliver cell fate reporters throughout spheroids, it is impossible to quantitatively assess treatment outcome. This uptake limitation is particularly problematic as large spheroids contain hypoxic cores that are known to be unresponsive to many therapies. The application of label-free imaging methods, such as endogenous multiphoton microscopy, OCT and photoacoustic tomography, may serve to address these issues in the future [46].

Given the importance of oxygen to the success of most PDT regimens, there is a significant need for improved methods of oxygen quantification within spheroids to visualize, map, and correlate microscale $\mathrm{pO}_{2}$ to treatment outcome. While technologies such as photoacoustic tomography are providing new insight into blood oxygen saturation levels during PDT in vivo, [47] such toolkits rely on the presence of blood and do not directly report tissue oxygen levels. Recent efforts developing porphyrin dendrimers capable of penetrating throughout 3D spheroid models show much promise for future PDT studies, as oxygen concentration can be mapped on the cellular level before, during, and after treatment [48]. This approach will likely become more easily applied with the advent of new, brighter porphyrin oxygen sensors.

The same simplicity that makes spheroid models useful also can limit their predictive ability. 3D tumor spheroids are avascular and only model uptake from the surrounding media or embedding matrix. As such, they are not likely to be of help in 
understanding therapies that induce vascular shutdown or that require the enhanced permeability and retention (EPR) effect for uptake. For example, the ovarian cancer spheroids developed by Hasan and colleagues are excellent at simulating small peritoneal metastases, but likely are not entirely informative in predicting PDT outcomes of vascularized metastatic lesions. More advanced models, which incorporate blood vessel-like structures are required to better assess such treatment approaches. A recent study investigating the effect of flow in ovarian $3 \mathrm{D}$ cultures hints at the promise of more advanced in vitro models [49]. Additionally, multicomponent spheroids, which incorporate cells such as tumor associated fibroblasts or endothelial cells are likely to play a role in simulating complex tumor microenvironments and heterogeneity [50]. Ultimately, there is a need to carry out sideby-side correlative studies directly comparing PDT outcomes

\section{References}

1. Licha K, Olbrich C. Optical imaging in drug discovery and diagnostic applications. Adv Drug Deliv Rev. (2005) 57:1087-108. doi: 10.1016/j.addr.2005.01.021

2. Vakoc BJ, Lanning RM, Tyrrell JA, Padera TP, Bartlett LA, Stylianopoulos T, et al. Three-dimensional microscopy of the tumor microenvironment in vivo using optical frequency domain imaging. Nat Med. (2009) 15:1219-23. doi: $10.1038 / \mathrm{nm} .1971$

3. Zhu TC, Finlay JC. Prostate PDT dosimetry. Photodiagnosis Photodyn Ther. (2006) 3:234-46. doi: 10.1016/j.pdpdt.2006.08.002

4. Fukumura D, Duda DG, Munn LL, Jain RK. Tumor microvasculature and microenvironment: novel insights through intravital imaging in preclinical models. Microcirculation (2010) 17:206-25. doi: 10.1111/j.15498719.2010.00029.x

5. Sutherland RM, McCredie JA, Inch WR. Growth of multicell spheroids in tissue culture as a model of nodular carcinomas. J Natl Cancer Inst. (1971) 46:113-20.

6. Friedrich J, Seidel C, Ebner R, Kunz-Schughart LA. Spheroid-based drug screen: considerations and practical approach. Nat Protoc. (2009) 4:309-24. doi: 10.1038/nprot.2008.226

7. Debnath J, Brugge JS. Modelling glandular epithelial cancers in threedimensional cultures. Nat Rev Cancer. (2005) 5:675-88. doi: 10.1038/nrc1695

8. Kim JB. Three-dimensional tissue culture models in cancer biology. Semin Cancer Biol. (2005) 15:365-77. doi: 10.1016/j.semcancer.2005.05.002

9. Kunz-Schughart LA, Freyer JP, Hofstaedter F, Ebner R. The use of 3-D cultures for high-throughput screening: the multicellular spheroid model. J Biomol Screen. (2004) 9:273-85. doi: 10.1177/1087057104265040

10. Kelm JM, Timmins NE, Brown CJ, Fussenegger M, Nielsen LK. Method for generation of homogeneous multicellular tumor spheroids applicable to a wide variety of cell types. Biotechnol Bioeng. (2003) 83:173-80. doi: 10.1002/bit.10655

11. Madsen SJ, Sun CH, Tromberg BJ, Cristini V, De Magalhães N, Hirschberg H. Multicell tumor spheroids in photodynamic therapy. Lasers Surg Med. (2006) 38:555-64. doi: 10.1002/lsm.20350

12. Dubessy C, Merlin JL, Marchal C, Guillemin F. Spheroids in radiobiology and photodynamic therapy. Crit Rev Oncol Hematol. (2000) 36:179-92. doi: 10.1016/S1040-8428(00)00085-8

13. Hirschberg H, Sun CH, Tromberg BJ, Madsen SJ. ALA-and ALA-estermediated photodynamic therapy of human glioma spheroids. J Neurooncol. (2002) 57:1-7. doi: 10.1023/A:1015784926550

14. Madsen SJ, Sun CH, Tromberg BJ, Hirschberg H. Repetitive 5-aminolevulinic acid-mediated photodynamic therapy on human glioma spheroids. $J$ Neurooncol. (2003) 62:243-250. doi: 10.1023/A:1023362011705

15. Madsen SJ, Sun CH, Tromberg BJ, Yeh AT, Sanchez R, Hirschberg H. Effects of combined photodynamic therapy and ionizing radiationon human glioma spheroids. Photochem Photobiol. (2002) 76:411-6. doi: 10.1562/00318655(2002)076<0411:EOCPTA > 2.0.CO;2 in spheroid cultures to those in animal models to examine the predictive capability of in vitro cultures. This will be important to carry out not only in immunocompromised cell-line and patient-derived xenograft animal models, but also in immunocompetent animals to assess the degree of immune system involvement.

\section{Author Contributions}

CE wrote the text of this manuscript.

\section{Acknowledgments}

We thank Mei Rosa Ng for her help in the editing of this manuscript.

16. Celli JP, Rizvi I, Evans CL, Abu-Yousif AO, Hasan T. Quantitative imaging reveals heterogeneous growth dynamics and treatment-dependent residual tumor distributions in a three-dimensional ovarian cancer model. J Biomed Opt. (2010) 15:051603. doi: 10.1117/1.3483903

17. Glidden MD, Celli JP, Massodi I, Rizvi I, Pogue BW, Hasan T. Imagebased quantification of benzoporphyrin derivative uptake, localization, and photobleaching in 3D tumor models, for optimization of PDT parameters. Theranostics (2012) 2:827. doi: 10.7150/thno.4334

18. Celli JP, Rizvi I, Blanden AR, Massodi I, Glidden MD, Pogue BW, et al. An imaging-based platform for high-content, quantitative evaluation of therapeutic response in 3D tumour models. Sci Rep. (2014) 4:3751. doi: 10.1038 /srep03751

19. Etminan N, Peters C, Ficnar J, Anlasik S, Bnemann E, Slotty PJ, et al. Modulation of migratory activity and invasiveness of human glioma spheroids following 5-aminolevulinic acidbased photodynamic treatment. J Neurosurg. (2011) 115:281-8. doi: 10.3171/2011.3.JNS10434

20. Jiang F, Chopp M, Katakowski M, Cho K, Yang X, Hochbaum N, et al. Photodynamic therapy with photofrin reduces invasiveness of malignant human glioma cells. Lasers Med Sci. (2002) 17:280-8. doi: 10.1007/s1010302 00041

21. Hirschberg H, Sun CH, Krasieva T, Madsen SJ. Effects of ALA-mediated photodynamic therapy on the invasiveness of human glioma cells. Lasers Surg Med. (2006) 38:939-45. doi: 10.1002/lsm.20445

22. Evans CL, Rizvi I, Celli J, Abu-Yousif A, de Boer J, Hasan T. Visualizing photodynamic therapy response with time-lapse OCT in an in vitro model of metastatic ovarian cancer. In: BiOS. San Francisco, CA: International Society for Optics and Photonics (2010). p. 75510J. doi: 10.1117/12.843271

23. Evans CL, Abu-Yousif AO, Park YJ, Klein OJ, Celli JP, Rizvi I, et al. Killing hypoxic cell populations in a 3D tumor model with EtNBS-PDT. PLoS ONE (2011) 6:e23434. doi: 10.1371/journal.pone.0023434

24. Klein O, Jung Y, Evans C. Longitudinal, quantitative monitoring of therapeutic response in $3 \mathrm{D}$ in vitro tumor models with OCT for high-content therapeutic screening. Methods (2014) 66:299-311. doi: 10.1016/j.ymeth.2013.08.028

25. Madsen SJ, Sun CH, Tromberg BJ, Wallace VP, Hirschberg $H$ Photodynamic therapy of human glioma spheroids using 5aminolevulinic acid. Photochem Photobiol. (2000) 72:128-34. doi: 10.1562/0031-8655(2000)072<0128:PTOHGS $>2.0$. CO;2

26. Mathews MS, Angell-Petersen E, Sanchez R, Sun CH, Vo V, Hirschberg H, et al. The effects of ultra low fluence rate single and repetitive photodynamic therapy on glioma spheroids. Lasers Surg Med. (2009) 41:578-84. doi: $10.1002 / \mathrm{lsm} .20808$

27. Bigelow C, Mitra S, Knuechel R, Foster T. ALA-and ALA-hexylesterinduced protoporphyrin IX fluorescence and distribution in multicell tumour spheroids. Br J Cancer. (2001) 85:727. doi: 10.1054/bjoc.2001.1977

28. Xiao Z, Hansen CB, Allen TM, Miller GG, Moore RB. Distribution of photosensitizers in bladder cancer spheroids: implications for intravesical instillation of photosensitizers for photodynamic therapy of bladder cancer. J Pharm Pharm Sci. (2005) 8:536-43. 
29. Klein OJ, Bhayana B, Park YJ, Evans CL. In vitro optimization of EtNBSPDT against hypoxic tumor environments with a tiered, high-content, 3D model optical screening platform. Mol Pharm. (2012) 9:3171-82. doi: $10.1021 / \mathrm{mp} 300262 \mathrm{x}$

30. Georgakoudi I, Nichols MG, Foster TH. The mechanism of photofrin photobleaching and its consequences for photodynamic dosimetry. Photochem Photobiol. (1997) 65:135-44. doi: 10.1111/j.1751-1097.1997.tb01889.x

31. Georgakoudi I, Foster TH. Singlet oxygen-versus nonsinglet oxygen-mediated mechanisms of sensitizer photobleaching and their effects on photodynamic dosimetry. Photochem Photobiol. (1998) 67:612-25. doi: 10.1111/j.17511097.1998.tb09102.x

32. Coutier S, Mitra S, Bezdetnaya LN, Parache RM, Georgakoudi I, Foster $\mathrm{TH}$, et al. Effects of fluence rate on cell survival and photobleaching in meta-tetra-(hydroxyphenyl)chlorinphotosensitized colo 26 multicell tumor spheroids. Photochem Photobiol. (2001) 73:297-303. doi: 10.1562/00318655(2001)073<0297:EOFROC>2.0.CO;2

33. Finlay JC, Mitra S, Patterson MS, Foster TH. Photobleaching kinetics of photofrin in vivo and in multicell tumour spheroids indicate two simultaneous bleaching mechanisms. Phys Med Biol. (2004) 49:4837. doi: 10.1088/00319155/49/21/001

34. Foster TH, Hartley DF, Nichols MG, Hilf R. Fluence rate effects in photodynamic therapy of multicell tumor spheroids. Cancer Res. (1993) 53:1249-54.

35. Nichols M, Foster T. Oxygen diffusion and reaction kinetics in the photodynamic therapy of multicell tumour spheroids. Phys Med Biol. (1994) 39:2161. doi: 10.1088/0031-9155/39/12/003

36. Mitra S, Foster TH. Photophysical parameters, photosensitizer retention and tissue optical properties completely account for the higher photodynamic efficacy of meso-tetra-hydroxyphenyl-chlorin vs photofrin. Photochem Photobiol. (2005) 81:849-59. doi: 10.1562/2005-02-22-RA-447R.1

37. West C. Size-dependent resistance of human tumour spheroids to photodynamic treatment. Br J Cancer. (1989) 59:510. doi: 10.1038/bjc.1989.105

38. West CM, Moore JV. Mechanisms behind the resistance of spheroids to photodynamic treatment: a flow cytometry study. Photochem Photobiol. (1992) 55:425-30. doi: 10.1111/j.1751-1097.1992.tb04257.x

39. Kamuhabwa AR, Huygens A, Roskams T, De Witte PA. Enhancing the photodynamic effect of hypericin in human bladder transitional cell carcinoma spheroids by the use of the oxygen carrier, perfluorodecalin. Int J Oncol. (2006) 28:775-80. doi: 10.3892/ijo.28.3.775

40. Huygens A, Kamuhabwa AR, Van Laethem A, Roskams T, Van Cleynenbreugel B, Van Poppel $\mathrm{H}$, et al. Enhancing the photodynamic effect of hypericin in tumour spheroids by fractionated light delivery in combination with hyperoxygenation. Int J Oncol. (2005) 26:1691-7. doi: 10.3892/ijo.26. 6.1691

41. Zelenkov P, Baumgartner R, Bise K, Heide M, Meier R, Stocker S, et al. Acute morphological sequelae of photodynamic therapy with 5 -aminolevulinic acid in the C6 spheroid model. J Neurooncol. (2007) 82:49-60. doi: 10.1007/s11060006-9252-8

42. Hirschberg H, Sun CH, Tromberg BJ, Yeh AT, Madsen SJ. Enhanced cytotoxic effects of 5-aminolevulinic acid-mediated photodynamic therapy by concurrent hyperthermia in glioma spheroids. J Neurooncol. (2004) 70:289-99. doi: 10.1007/s11060-004-9161-7

43. Purkiss S, Grahn M, Turkish M, Macey M, Williams N. In vitro modulation of haematoporphyrin derivative photodynamic therapy on colorectal carcinoma multicellular spheroids by verapamil. Br J Surg. (1992) 79:120-5. doi: 10.1002/bjs.1800790209

44. Rizvi I, Celli JP, Evans CL, Abu-Yousif AO, Muzikansky A, Pogue BW, et al. Synergistic enhancement of carboplatin efficacy with photodynamic therapy in a three-dimensional model for micrometastatic ovarian cancer. Cancer Res. (2010) 70:9319-28. doi: 10.1158/0008-5472.CAN-10-1783

45. Alderden RA, Mellor HR, Modok S, Hall MD, Sutton SR, Newville MG, et al. Elemental tomography of cancer-cell spheroids reveals incomplete uptake of both platinum (II) and platinum (IV) complexes. J Am Chem Soc. (2007) 129:13400-1. doi: 10.1021/ja076281t

46. Jung Y, Nichols AJ, Klein OJ, Roussakis E, Evans CL. Label-free, longitudinal visualization of PDT response in vitro with optical coherence tomography. Isr J Chem. (2012) 52:728-44. doi: 10.1002/ijch.201200009

47. Mallidi S, Huang HC, Liu JY, Mensah L, Mai Z, Hasan T. Photoacoustic image guided photodynamic therapy of glioblastoma. Cancer Res. (2013) 73(8 Suppl.):3923. doi: 10.1158/1538-7445.AM2013-3923

48. Nichols AJ, Roussakis E, Klein OJ, Evans CL. Click-assembled, oxygen-sensing nanoconjugates for depth-resolved, near-infrared imaging in a $3 \mathrm{D}$ cancer model. Angew Chem Int Ed. (2014) 53:3671-4. doi: 10.1002/anie.201311303

49. Rizvi I, Gurkan UA, Tasoglu S, Alagic N, Celli JP, Mensah LB, et al. Flow induces epithelial-mesenchymal transition, cellular heterogeneity and biomarker modulation in 3D ovarian cancer nodules. Proc Natl Acad Sci USA. (2013) 110:E1974-83. doi: 10.1073/pnas.1216989110

50. Xu F, Celli J, Rizvi I, Moon S, Hasan T, Demirci U. A three-dimensional in vitro ovarian cancer coculture model using a high-throughput cell patterning platform. Biotechnol J. (2011) 6:204-12. doi: 10.1002/biot.201000340

Conflict of Interest Statement: The author declares that the research was conducted in the absence of any commercial or financial relationships that could be construed as a potential conflict of interest.

Copyright (c) 2015 Evans. This is an open-access article distributed under the terms of the Creative Commons Attribution License (CC BY). The use, distribution or reproduction in other forums is permitted, provided the original author(s) or licensor are credited and that the original publication in this journal is cited, in accordance with accepted academic practice. No use, distribution or reproduction is permitted which does not comply with these terms. 